\title{
Gestión del conocimiento en el marco de la innovación en la Facultad de Ciencias Juridicas de la Universidad de Manizales: Auditoria de conocimiento*
}

\author{
Knowledge Management within the Framework of Innovation in the Faculty of Legal Sciences of the University of \\ Manizales: Knowledge Audit
}

Camilo González Carreño ${ }^{a}$

Universidad de Manizales, Colombia

DOI: https://doi.org/10.11144/Javeriana.vj139.gcmi

camilo.gonzalezca@amigo.edu.co

ORCID: http://orcid.org/0000-0002-7651-0336

Recepción: 28 Noviembre 2018

Aceptación: 29 Junio 2019

Mónica Cecilia Montoya Escobar

Fecha de publicación: 30 Diciembre 2019

Universidad Autónoma Latinoamericana, Colombia

ORCID: http://orcid.org/0000-0001-8429-4656

\section{Resumen:}

El aporte a la optimización de la gestión del conocimiento en el Programa de Derecho de la Facultad de Ciencias Jurídicas de la Universidad de Manizales emerge como interés primordial en este estudio, y puede evidenciarse a través de la formulación de un plan de acción diseñado por los autores, luego de determinar el estado de la gestión del conocimiento de dicho programa mediante la implementación de una metodología de auditoría del conocimiento que se basó en el enfoque de los procesos clave de la organización. Se desarrolla bajo un enfoque empírico-analítico, cuyo nivel de análisis se identificó en dos momentos: descriptivo y propositivo; este último consistió en el plan de acción como aporte a la planeación estratégica. El plan de acción está compuesto de acciones de mejora ubicadas estratégicamente, de acuerdo a los procesos de la gestión del conocimiento: adquisición, difusión y aplicación, pero también enmarcadas dentro de la innovación educativa. El surgimiento de las acciones de mejora se dio a partir de las brechas de conocimiento existentes y detectadas. Este análisis, con ánimo propositivo, permitió contribuir a la planificación estratégica del programa con miras a sostener los estándares de calidad misional con los que se identifica. Así, se concluye que los principales factores de éxito en la gestión del conocimiento del programa intervenido son la alineación entre las políticas institucionales y la gestión administrativa del programa, junto con la existencia de escenarios y espacios que promuevan la comunicación directa y el intercambio de conocimiento entre los diversos actores ligados al mismo. Asimismo, las brechas o aspectos por mejorar se encuentran en la poca experiencia de un grupo de docentes del programa que aún tienen carencias en la formación sobre procesos críticos, en especial en materia de investigación y el escaso uso de las TIC para documentar y compartir el conocimiento dentro y fuera de la institución.

Palabras clave: gestión del conocimiento, auditoría de conocimiento, innovación educativa, institución de educación superior.

\section{Abstract:}

The contribution to the optimization of knowledge management in the Law Program of the Faculty of Legal Sciences of the University of Manizales emerges as a primary interest in this study and can be evidenced through the formulation of an action plan designed by the authors, after determining the state of knowledge management of the program through the implementation of a knowledge audit methodology based on the focus of the organization's key processes. It is developed under an empirical-analytical approach, whose level of analysis was identified in two moments: descriptive and proactive; the last one consisted of the action plan as a contribution to strategic planning. The action plan is composed of improvement actions strategically located according to the processes of knowledge management: acquisition, dissemination, and application, but also framed in educational innovation. The emergence of improvement actions was based on existing and detected knowledge gaps. Its analysis with a proactive intention, allowed with this study, to contribute to the strategic planning of the program to sustain the missional quality standards with which it is identified. Thus, it is concluded that the main factors of success in managing knowledge of the operated program are the alignment between institutional policies and administrative management of the program, together with the existence of scenarios and spaces that promote direct communication and exchange of knowledge among the various actors linked to it. Also, gaps or areas for improvement found in the lack of experience of a group of teachers from the program that still have gaps in training on critical processes, especially in research and limited use of ICT to document and share the knowledge inside and outside the institution.

Notas de autor

\footnotetext{
${ }^{a}$ Autor de correspondencia. Correo electrónico: camilo.gonzalezca@amigo.edu.co
} 
Keywords: knowledge management, knowledge audit, educational innovation, higher educational institutions.

\section{Introducción}

Con el propósito de aportar a la optimización de la gestión del conocimiento como insumo básico de la innovación educativa, específicamente en el Programa de Derecho de la Universidad de Manizales, se trazó un plan de acción cuya ejecución sería benéfica ${ }^{[1]}$, puesto que las acciones propuestas se diseñaron pensando en facilitarle a la comunidad interna y externa del Programa de Derecho el acceso al conocimiento a través de una estructura formal.

El logro de dicho cometido empezó con la lectura y análisis de documentos sobre modelos y metodologías de auditorías de conocimiento, lo que permitió seleccionar y adaptar una metodología sobre la dinámica de gestión académica y administrativa que el Programa de Derecho ya venía reconociendo.

La metodología seleccionada fue la planteada en 2006 por docentes investigadores de la ciudad de México, en cuya cabeza se reconoce a Alonso Pérez-Soltero, llamada "Metodología con énfasis en los procesos clave".

Una de las razones para seleccionar esta propuesta metodológica entre las ocho estudiadas es que fue aplicada por sus creadores en la Universidad Veracruzana, región Xalapa, lo cual tiene especial afinidad con el objeto de estudio y el contexto académico latinoamericano. Además, llamó la atención el hecho de que reconoce como crítico el activo de conocimiento que poseen las personas y que se centra en los procesos clave de la organización (llamados así porque contienen las actividades fundamentales para el éxito de la organización); taxonomía que sigue la línea de las instituciones de educación superior colombianas, al considerar como procesos centrales o misionales la docencia, la investigación, la proyección social y la gestión académica y administrativa.

La implementación de las primeras fases de la metodología permitió identificar los procesos críticos y las personas claves en el programa. Con el desarrollo de las siguientes, se logró establecer el estado de la gestión del conocimiento en el Programa de Derecho de la Facultad de Ciencias Jurídicas, gracias a la construcción de las siguientes herramientas: inventario de conocimiento, flujo de conocimiento y mapa de conocimiento. Estas herramientas permitieron visualizar la circulación del conocimiento en el programa respecto a su adquisición, difusión y aplicación.

Finalmente, gracias a los datos obtenidos mediante los instrumentos aplicados como exigencia de la metodología adoptada surgieron brechas de mejora y factores de éxito en cada proceso, que fueron la base del plan de acción y cuya construcción motivó este estudio. La investigación utilizó una metodología mixta que comprende un análisis desde lo cuantitativo y lo cualitativo con un enfoque empírico-analítico. El nivel de análisis se identificó en dos momentos: descriptivo y propositivo, este último consistió en el plan de acción como aporte a la planeación estratégica.

Tras la realización del estudio, se hizo evidente que para iniciar una estrategia de gestión del conocimiento en una organización se debe comenzar por realizar una auditoría de conocimiento, con el fin de identificar las brechas existentes y usar los resultados como base para el desarrollo de un plan de acción que permita la disminución de dichas brechas. Además, es importante reconocer que la selección de la metodología de auditoría de conocimiento debe proporcionar una ruta apropiada de acuerdo con el contexto educativo. Para el caso de un programa educativo en una institución de educación superior, la mejor selección es aquella que ya ha sido aplicada en otros programas de instituciones educativas más allá de la organización empresarial, ya que el conocimiento de las personas es el activo principal de cualquier IES.

Luego de analizar los resultados de la auditoría realizada, se concluye que los principales factores de éxito en la gestión del conocimiento son la alineación entre las políticas institucionales y la gestión administrativa del programa, junto con la existencia de escenarios y espacios que promuevan la comunicación directa y el intercambio de conocimiento entre los diversos actores ligados al mismo. Asimismo, las brechas o aspectos 
débiles por mejorar se encuentran en la poca experiencia de un grupo de docentes del programa que aún tienen carencias en la formación sobre procesos críticos, en especial en materia de investigación y el escaso uso de las TIC para documentar y compartir el conocimiento dentro y fuera de la institución.

\section{Diseño Metodológico}

La investigación se realizó en varias etapas: revisión teórica, marco conceptual (que se concentró en el estudio de los siguientes campos semánticos: conocimiento ${ }^{[2]}$, gestión del conocimiento ${ }^{[3]}$, innovación y auditoría de conocimiento $^{[4]}$, definición de técnicas y elaboración de instrumentos. A partir de este ejercicio, se obtuvo la información necesaria para respondera la pregunta central de la investigación: ¿Cómo contribuir con el mejoramiento de los procesos académicos y administrativos del programa de derecho de la Universidad de Manizales en el marco de la gestión del conocimiento y la innovación?

\section{Tipo de estudio}

La investigación fue de corte mixto (cuantitativo-cualitativo con un enfoque empírico-analítico, en tanto se apoyó en herramientas de corte cualitativo como entrevistas y grupo focal; estas herramientas se aplicaron para soportar la lectura de los hallazgos que propusieron los instrumentos de tipo cuantitativo como las encuestas a los diferentes actores participantes a quienes se les aplicaron. Se pretende, entonces, describir y explicar cómo se gestiona el conocimiento en el Programa de Derecho de la Facultad de Ciencias Jurídicas de la Universidad de Manizales, a través de la observación a sus procesos clave sobre el conocimiento, que es el tema principal del estudio. Sumado a ello, se realizó un análisis que permitiera identificar aspectos como el conocimiento con que se cuenta al momento de realizar el estudio, cómo se crea, cómo fluye, cómo se transmite y cuál es su desempeño. Con este análisis, se buscó conocer la experiencia de los actores en el manejo y la gestión del conocimiento. El alcance de este estudio tuvo dos momentos:

\section{Momento descriptivo}

En este momento se compilaron documentos referentes a la gestión del conocimiento ${ }^{[5]}$, la innovación y la auditoría del conocimiento ${ }^{[6]}$. A continuación, se aplicó una metodología de auditoría de conocimiento que permitiera establecer y describir el estado actual de la gestión del conocimiento en el programa, resultados que llevan al segundo momento.

\section{Momento propositivo}

Este momento consistió en la formulación de un plan de acción que aportara a la planeación estratégica del programa y permitiera optimizar la gestión del conocimiento como insumo básico de la innovación educativa en el Programa de Derecho de la Facultad de Ciencias Jurídicas de la Universidad de Manizales.

\section{Caso de Estudio}

La investigación se llevó a cabo en el Programa de Derecho de la Facultad de Ciencias Jurídicas de la Universidad de Manizales. Este programa está acreditado en alta calidad desde el 6 de septiembre de $2012^{[7]}$. El título otorgado es el de abogado. Tiene una duración estimada de 10 períodos académicos, con 161 créditos. 
Su modalidad de formación es completamente presencial y se ofrece en jornada diurna y nocturna. Está adscrito a la Facultad de Ciencias Jurídicas desde la creación misma de la facultad, hace 44 años. Cuenta con 1.110 estudiantes, 67 profesores, y atiende en promedio en el consultorio jurídico 350 usuarios al mes.

\section{Resultados}

\section{Estado de la gestión del conocimiento en el Programa de Derecho de la Facultad de Ciencias Jurídicas}

Esta propuesta proviene de la aplicación del modelo de auditoría desarrollado por Alonso Pérez-Soltero, Mario Barcelo-Valenzuela, Gerardo Sánchez-Schmitz, Fernando Martín-Rubio, José Tomás Palma-Méndez y Adolfo Alberto Vanti, que se centra en el enfoque de los procesos clave; llamados así porque contienen las actividades apropiadas para el éxito de la organización, tanto que, el no realizarlas da lugar al incumplimiento de la misión organizacional ${ }^{[8]}$.

A continuación, se exponen las etapas del modelo que se adaptan a la dinámica del Programa de Derecho y de la Facultad a la que está adscrito. Para el presente artículo únicamente se hará la presentación de cada una de las etapas que fueron analizadas durante el proceso investigativo.

\section{Etapa 1. Información estratégica del programa y procesos de la organización}

El primer objetivo Consistió en adquirir información estratégica del programa, que permitiera identificar su misión, visión y procesos clave. Esta etapa inició con las entrevistas aplicadas a la decana de la Facultad de Ciencias Jurídicas, a quien se le presentaron los objetivos del proyecto y los conceptos de gestión del conocimiento y de auditoría de conocimiento, para ubicar los activos de conocimiento y el flujo de estos en la organización.

Se observa que la administración del programa concibe la gestión del conocimiento en sinergia con la innovación, que parte de un conocimiento aplicado (transformado, según la entrevistada) y transformador (que impacte la realidad). Esto es relevante porque "la innovación no puede ser una práctica aislada, sino por el contrario, debe responder a una necesidad de diferenciación estratégica" ${ }^{[9]}$.

\section{Etapa 2. Procesos del Programa de Derecho y criterios de medición}

El segundo objetivo consistió en identificar los procesos claves del programa que contienen conocimientos útiles para ser administrados, y las herramientas para medir el rendimiento de los procesos de conocimiento.

Los procesos clave son un conjunto de actividades con diversas funciones que son esenciales para la satisfacción del cliente externo y para el logro de la misión de la organización. A estas actividades se integran las personas, materiales, energía, equipos e información ${ }^{[10]}$. De manera que, identificados los procesos clave de la organización, se establece dónde existe el conocimiento valioso y en manos de quién está, para luego hacer su valoración y clasificación.

De este análisis, se establece la importancia de los procesos académicos y administrativos en la organización, que se consideran sustanciales según la prestación del servicio educativo y la gestión del conocimiento.

Por último, se contemplan las siguientes características enunciadas por Alonso Pérez Soltero et al. en el año 2008, como un filtro que permite seleccionar los procesos del programa:

- Tienen impacto directo con la misión y visión. 
- Son críticas para el éxito general de la organización.

- Tienen impacto y le dan un valor añadido a la organización.

- Permiten satisfacer los requisitos del cliente.

- Cuentan con recursos humanos, tecnológicos y de información valiosa.

\section{Etapa 3. Seleccionar los procesos clave de la organización}

El objetivo de esta etapa fue priorizar los procesos de la organización de acuerdo con las herramientas de soporte enunciadas en la etapa 2.

Para priorizar los procesos, se les pregunta a los líderes por la información documental interna y externa del programa, de la Facultad a la que pertenece y de la Universidad. La información documental a la que se hace referencia y que fue utilizada para esta investigación, está incluida en el reglamento estudiantil, el estatuto docente, el reglamento interno de trabajo, el reglamento del consultorio jurídico y el centro de conciliación, el PEP (Proyecto Educativo del Programa), los estatutos de la universidad, el modelo de autoevaluación, el reglamento de investigaciones, el modelo pedagógico institucional, el plan de acción vigente, el modelo curricular y la resolución de acreditación en alta calidad.

De la aplicación y el análisis de las herramientas soporte se establecen como procesos del Programa de Derecho la docencia, la investigación, la proyección social y la gestión académica y administrativa. Esta selección permitió comprobar la construcción epistemológica y las prácticas de cómo se hace efectiva la gestión curricular, las entradas, las salidas, los proveedores de información y clientes directos del proceso ${ }^{[11]}$.

\section{Etapa 4. Identificar las personas clave}

El objetivo de esta etapa fue el de identificar a las personas que participan en los procesos clave seleccionados.

Las personas clave en el proceso son los docentes y los líderes funcionales que administran los procesos, lo cual se ratifica con la revisión de sus perfiles: docentes directos, investigadores, asesores de consultorio y líderes funcionales. Se identifican entonces como líderes de los procesos a la coordinadora de programa por el proceso de docencia; a la coordinadora de investigaciones por el proceso de investigación; al director del consultorio jurídico y la coordinadora del centro de conciliación, por el proceso de proyección social; y a la decana por el proceso de gestión administrativa y académica.

\section{Etapa 5. Reunión con personas clave}

El objetivo de esta etapa fue el de dar información a las personas clave sobre la auditoría del conocimiento y los procesos de gestión del conocimiento.

En reunión preliminar se puso al tanto del proceso de auditoría a una muestra representativa de los docentes del programa ( 27 de 67 docentes que conforman la planta) y a los líderes funcionales de los procesos clave, mencionados en la etapa anterior. Se les explica el concepto de gestión de conocimiento y el de auditoría de conocimiento para que ellos puedan reconocer y aportar al inventario de conocimiento y al flujo de conocimiento propios de la auditoría.

\section{Etapa 6. Obtención de inventario de conocimiento}

El objetivo de esta etapa fue el de localizar y obtener los activos de conocimiento existentes dentro del programa. 
Tras la recolección de la información de las diversas fuentes mediante entrevistas, grupo focal, encuestas, revisión documental y el posterior análisis de ésta, se elaboró el siguiente inventario de conocimiento (ver tabla 1), concepto que aduce a la localización y obtención de los activos del conocimiento existentes en el programa.

TABLA 1.

Inventario de conocimiento

\begin{tabular}{|c|c|c|c|c|c|}
\hline Procesos & $\begin{array}{c}\text { Ittem de } \\
\text { cobocimbento (Temm } \\
\text { clave) }\end{array}$ & $\begin{array}{c}\text { Persona (s) que bo } \\
\text { poseen }\end{array}$ & $\begin{array}{c}\text { Fintidades u } \\
\text { organizaciones que } \\
\text { bo poseen }\end{array}$ & $\begin{array}{l}\text { Sistema donde se } \\
\text { encuentra }\end{array}$ & $\begin{array}{l}\text { Docume ntos donde } \\
\text { se encientru }\end{array}$ \\
\hline \multirow{5}{*}{ Proyecebion Social } & $\begin{array}{l}\text { Briaslas de } \\
\text { imenencion a ba } \\
\text { comandad }\end{array}$ & $\begin{array}{l}\text { Docentes asesores, } \\
\text { Estudiantes } \\
\text { practicames, docentes } \\
\text { conciladores }\end{array}$ & & $\begin{array}{l}\text { Portal web de ha } \\
\text { faculad }\end{array}$ & \\
\hline & $\begin{array}{l}\text { Represctsacxint a } \\
\text { usturios en ltivio }\end{array}$ & $\begin{array}{l}\text { Estudiantes } \\
\text { practicamies }\end{array}$ & Rama juaticial & $\begin{array}{l}\text { Pottal web de h rana } \\
\text { jucicinl }\end{array}$ & $\begin{array}{l}\text { Reglameno de } \\
\text { contsutworio juridioo. } \\
\text { expedientes de } \\
\text { represemaciva }\end{array}$ \\
\hline & $\begin{array}{l}\text { Audiencias de } \\
\text { coscilacion }\end{array}$ & Docentes concliadores & & $\begin{array}{l}\text { Portal web de h } \\
\text { ficulad, SICAAC } \\
\text { (Mujutixixi) }\end{array}$ & $\begin{array}{l}\text { Maminl de } \\
\text { compelencias y } \\
\text { fincianes del centro de } \\
\text { concilacion, anchinos } \\
\text { Ad centro de } \\
\text { concilación }\end{array}$ \\
\hline & Cinca Juridica & $\begin{array}{l}\text { Asesones de cfrica } \\
\text { jeridica }\end{array}$ & & $\begin{array}{l}\text { Portal web of h } \\
\text { faculad }\end{array}$ & \\
\hline & Eulucación contmada & $\begin{array}{l}\text { Dooctses, externos } \\
\text { ponemies }\end{array}$ & Universidades & $\begin{array}{l}\text { Portal web de b } \\
\text { foculad }\end{array}$ & Artictüs, rehtoras \\
\hline \multirow{3}{*}{ Investigación } & $\begin{array}{l}\text { Fonuncion } \\
\text { imestigntiva }\end{array}$ & $\begin{array}{l}\text { Coordinadores de } \\
\text { similkto, Elctes de } \\
\text { gnqo }\end{array}$ & $\begin{array}{l}\text { Cokinicis, reds de } \\
\text { investipucioin }\end{array}$ & $\begin{array}{l}\text { Portal web de h } \\
\text { unimersidad }\end{array}$ & Resoluciva rectoral \\
\hline & Investigaciön cientîica & $\begin{array}{l}\text { Docentes doctores y } \\
\text { magister }\end{array}$ & Cokiencis & $\begin{array}{l}\text { Portal web de } \\
\text { Cokienciss, portal } \\
\text { web de a uninersidad }\end{array}$ & $\begin{array}{l}\text { Publicaciones, } \\
\text { rephimentos de } \\
\text { investigacionss }\end{array}$ \\
\hline & $\begin{array}{l}\text { Melodologias de } \\
\text { inestizackín }\end{array}$ & $\begin{array}{l}\text { Docertes } \\
\text { investigndores }\end{array}$ & Redes de imestigacion & $\begin{array}{l}\text { Pottal web de h } \\
\text { universidad }\end{array}$ & $\begin{array}{l}\text { Reglamentos de } \\
\text { imestigaciones, lboros } \\
\text { sobre inestiacixon }\end{array}$ \\
\hline \multirow{3}{*}{ Docencia } & $\begin{array}{l}\text { Derecbo sustantivo y } \\
\text { dogenitico } \\
\text { (Cossecanicuto } \\
\text { disciplinar) }\end{array}$ & Docentes & Corte constuxional & $\begin{array}{l}\text { Bases de davos, portal } \\
\text { web de ha univesdad }\end{array}$ & 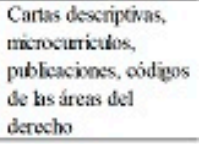 \\
\hline & $\begin{array}{l}\text { Metodobogis de } \\
\text { enseñanza y el } \\
\text { aprendizaje }\end{array}$ & $\begin{array}{l}\text { Doccutes, experwos } \\
\text { externos, Ederes } \\
\text { fiscionales }\end{array}$ & $\begin{array}{l}\text { Manislerio de } \\
\text { educacioin, } \\
\text { maversilades }\end{array}$ & $\begin{array}{l}\text { Portal web de h } \\
\text { uninersidad }\end{array}$ & $\begin{array}{l}\text { PEP, PIF, } \\
\text { microcuerictos }\end{array}$ \\
\hline & $\begin{array}{l}\text { Derodbo procesaly } \\
\text { procedmental } \\
\text { (Conocimiato } \\
\text { peíctixo) }\end{array}$ & $\begin{array}{l}\text { Docertes, experkos } \\
\text { extemos }\end{array}$ & $\begin{array}{l}\text { Manisleriv de juatich, } \\
\text { rama judicial }\end{array}$ & $\begin{array}{l}\text { Portal web de h rama } \\
\text { judicial, portal web del } \\
\text { miristerio de justicis, } \\
\text { portal web del senado } \\
\text { y portal web de h } \\
\text { universidad }\end{array}$ & 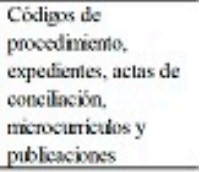 \\
\hline \multirow{3}{*}{ Gestión } & $\begin{array}{l}\text { Adnuinistraciōn de } \\
\text { unidades estratbigas }\end{array}$ & $\begin{array}{l}\text { Lideres fancionales, } \\
\text { decanatura }\end{array}$ & Universibades & $\begin{array}{l}\text { Portal web de h } \\
\text { uninersidad, SIGUM }\end{array}$ & $\begin{array}{l}\text { Regamnerios } \\
\text { institucionales, manual } \\
\text { de fitoxiones y perfics, } \\
\text { infirmes de gestionn }\end{array}$ \\
\hline & $\begin{array}{l}\text { Requistos de } \\
\text { acrodracion de ata } \\
\text { calidad }\end{array}$ & $\begin{array}{l}\text { Léderes finciomales, } \\
\text { decanatura y docentes }\end{array}$ & $\begin{array}{l}\text { Mnisterio de } \\
\text { clucacǘn, } \\
\text { universidades }\end{array}$ & $\begin{array}{l}\text { Portal web ministerio } \\
\text { de educacknt, porkal } \\
\text { weh de h taniversidad }\end{array}$ & 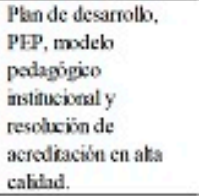 \\
\hline & Aucesaluciöin & $\begin{array}{l}\text { Figressios, } \\
\text { estudiantes, lideres } \\
\text { fircinnaks, decimatura } \\
\text { y docemles }\end{array}$ & $\begin{array}{l}\text { Manisierio de } \\
\text { educacixin, } \\
\text { wiversidades, gemios } \\
\text { econimios y } \\
\text { cooperantes en } \\
\text { ahanas estratégicas. }\end{array}$ & $\begin{array}{l}\text { Portal web de h } \\
\text { universidad, portal } \\
\text { web del nutisteris de } \\
\text { educación }\end{array}$ & $\begin{array}{l}\text { Modelo de } \\
\text { autcevalucicin }\end{array}$ \\
\hline
\end{tabular}

Fuente: elaboración propia 
A continuación, se examinará el inventario de conocimiento a la luz de los procesos clave previamente identificados en la auditoría del conocimiento. Este artículo presenta la síntesis de dicho análisis.

\section{Proceso clave proyección social}

La selección de los ítems de conocimiento o temas clave que caracteriza el proceso de Proyección en el Programa de Derecho (estos son: brigadas de intervención a la comunidad; representación a usuarios en litigio; audiencias de conciliación; clínica jurídica; educación continuada), surge del análisis de los datos que resultan de las respuestas de los encuestados (personas clave). Además, parte de una situación notoria y es que, en el Programa de Derecho de la Facultad de Ciencias Jurídicas, la relación con el sector externo y la proyección social del programa está concentrada en las labores de servicio social que prestan el consultorio jurídico y el centro de conciliación ${ }^{[12]}$.

En la tabla 2 se listan los factores de éxito identificados y las brechas de conocimiento detectadas con el análisis del inventario de conocimiento hecho al proceso de Proyección Social:

TABLA 2.

Proyección social

\begin{tabular}{|c|c|c|}
\hline \multicolumn{3}{|c|}{ Factores de éxito } \\
\hline Adquisición (almacenamiento) & Difusión & Aplicación (creación) \\
\hline \multirow{2}{*}{$\begin{array}{l}\text { Implementación de h norma NTC } 5906 \text { de } \\
2012 \text { en el centro de conciliación (pendientes } \\
\text { de ser certificados) }\end{array}$} & $\begin{array}{l}\text { Práctica académica: audiencias de conciliación, latigio, } \\
\text { asesorías juridicas, brigadas jurídicas }\end{array}$ & $\begin{array}{l}\text { Actividades de vohuntarado desde el consultorio } \\
\text { juridico y el centro de coneilación }\end{array}$ \\
\hline & $\begin{array}{l}\text { Diseño y ejecución de formación continua desde el proceso } \\
\text { chve, de manera independiente: montaje de diplomado en } \\
\text { conciliación y capactaciones externas en mediación }\end{array}$ & \\
\hline \multicolumn{3}{|c|}{ Brechas existentes } \\
\hline Adquisición (almacenamiento) & Difusión & Aplicación (creación) \\
\hline \multirow{2}{*}{$\begin{array}{l}\text { El desanollo de ba clinica juridica, que es } \\
\text { valorado por los estudiantes como neipiente. } \\
\text { Lo cual amerita llevar a cabo capacitaciones } \\
\text { en método clínico y litigio estratégico, así } \\
\text { como la amplación de cobertura de } \\
\text { asesores. }\end{array}$} & $\begin{array}{l}\text { A parte de los esfiucrzos bechos por el centro de } \\
\text { cone iliación, no se detecta un alto estínulo para ha } \\
\text { generación de programas de educación continua en estos } \\
\text { temas, y las cifras dan cuenta de ello, pues representa ba } \\
\text { rehación con el sector extemo: un } 5 \% \text { de los docentes usa los } \\
\text { eventos para compartir conocimiento con sus compañeros }\end{array}$ & $\begin{array}{l}\text { Se detecta poco fomento por parte de la Facultad } \\
\text { del trabajo cohborativo o en red (alianzas } \\
\text { estratégicas, convenios, comunidades de práctica, } \\
\text { participación en gremios o asociaciones, entre otras) } \\
\text { y de procesos de intemacionalización (moviliad, } \\
\text { intercambio de información). }\end{array}$ \\
\hline & $\begin{array}{l}\text { de trabajo; un } 10 \% \text { para compartir conocimiento con } \\
\text { estud rintes y otro } 10 \% \text { con terceros. Estos porcentajes son } \\
\text { significativamente bajos, si tenemos en cuenta que bs } \\
\text { eventos son una estrategia de proyección socivly de } \\
\text { apropiación socinl del conocimiento. }\end{array}$ & $\begin{array}{l}\text { Desde el centro de conciliación reconocen no saber } \\
\text { cómo atender personas con discapacidad. }\end{array}$ \\
\hline
\end{tabular}

Fuente: elaboración propia 


\section{Proceso clave docencia}

Los temas clave que caracteriza el proceso de docencia en el Programa de Derecho investigado, son el derecho sustantivo y dogmático; metodologías de enseñanza y de aprendizaje; derecho procesal y procedimental.

Es común a todas las fuentes consultadas comprender que la docencia como proceso clave, pues es una de las razones de ser de las universidades. Al respecto, Nonaka ${ }^{[13]}$ expresó, con relación a las organizaciones educativas, que el conjunto de conocimientos y saberes que son capaces de desarrollar constituye su principal fuente de progreso y el condicionante fundamental para su evolución futura.

Desde esta perspectiva, se evidencia que gran parte de la labor que realiza la comunidad académica del programa de derecho está direccionada a la gestión curricular, propia de la función sustantiva de docencia, pero que irradia el quehacer formador en todas sus dimensiones.

En la tabla 3 se relacionan los factores de éxito identificados y las brechas de conocimiento detectadas con el análisis detallado del inventario de conocimiento hecho al proceso clave de Docencia:

TABLA 3.

Docencia

\begin{tabular}{|c|c|c|}
\hline \multicolumn{3}{|c|}{ Factores de éxito } \\
\hline Adquisición (almacenamiento) & Difusión & Aplicación (creación) \\
\hline $\begin{array}{l}\text { Óptimo desarrollo de las asignaturas } \\
\text { prácticas del plan de estudios }\end{array}$ & & $\begin{array}{l}\text { Estimulo de prácticas tempranas } \\
\text { desde la articulación de la práctica y } \\
\text { la teoría }\end{array}$ \\
\hline \multicolumn{3}{|c|}{ Brechas existentes } \\
\hline Adquisición (almacenamiento) & Difusión & Aplicación (creación) \\
\hline \multirow{2}{*}{$\begin{array}{l}\text { Los microcurrículos suelen estar muy } \\
\text { desactualizados, lo cual no } \\
\text { corresponde a un currículo } \\
\text { responsable en tanto el derecho se } \\
\text { actualiza dia tras dia a partir del } \\
\text { análisis de temas de tendencia. Si no } \\
\text { se revisan las nuevas tendencias no se } \\
\text { puede hablar de un currículo } \\
\text { innovador. }\end{array}$} & $\begin{array}{l}\text { No existe un manejo frecuente de los } \\
\text { medios escritos, de los foros y la base } \\
\text { de datos, solo es usada por el } 15 \% \text { de } \\
\text { los docentes encuestados. Lo cual } \\
\text { permite concluir que la Facultad no } \\
\text { cuenta con un repositorio de carácter } \\
\text { institucional. }\end{array}$ & $\begin{array}{l}\text { Los docentes tienen poca experiencia } \\
\text { sobre trabajo colaborativo parece } \\
\text { primar el interés individual en la } \\
\text { realización de sus labores para } \\
\text { alcanzar efectividad por encima del } \\
\text { interés colectivo. }\end{array}$ \\
\hline & $\begin{array}{l}\text { Poco fomento a redes de } \\
\text { conocimiento. }\end{array}$ & \multirow{3}{*}{$\begin{array}{l}\text { La mayoría de docentes no utilizan } \\
\text { estrategias de enseñanza y } \\
\text { aprendizaje donde se articule la } \\
\text { práctica y la teoría, ello solo se ve en } \\
\text { las prácticas tempranas con } \\
\text { estudiantes de segundo semestre. }\end{array}$} \\
\hline $\begin{array}{l}\text { Faltan estrategias de documentación } \\
\text { de experiencias de encuentros entre } \\
\text { pares y expertos, y de los resultados } \\
\text { obtenidos por las labores } \\
\text { encomendadas. }\end{array}$ & \multirow[t]{2}{*}{$\begin{array}{l}\text { Los docentes del programa prefieren } \\
\text { las fuentes o medios humanos } \\
\text { (reuniones formales e informales), } \\
\text { para adquirir y difundir el } \\
\text { conocimiento, que los fisicos o } \\
\text { tecnológicos. }\end{array}$} & \\
\hline $\begin{array}{l}\text { Falta experticia en pedagogía del } \\
\text { derecho. }\end{array}$ & & \\
\hline
\end{tabular}

Fuente: elaboración propia 


\section{Proceso clave investigación}

Los ítems de conocimiento o temas críticos que caracterizan el proceso de investigación en el Programa de Derecho son la formación investigativa, la investigación científica y las metodologías de investigación.

Lo anterior surge del análisis de los datos de las respuestas de los encuestados (personas clave) y bajo el entendido de que la investigación cientifica o en formación origina un adecuado acercamiento a la gestión del conocimiento creado, adquirido y proporcionado en el campo académico. La tabla 4 muestra los factores de éxito y las brechas existentes en esta categoría.

TABLA 4.

Investigación

Factores de éxito

\begin{tabular}{|c|c|c|}
\hline Adquisición (almacenamiento) & Difusión & Aplicación (creación) \\
\hline \multirow{3}{*}{$\begin{array}{l}\text { Hay un repositorio de los soportes } \\
\text { de la productividad que está } \\
\text { manejado por los lideres de grupo a } \\
\text { través de la plataforma ScienTI de } \\
\text { Colciencias. }\end{array}$} & & $\begin{array}{l}\text { Apoyo a la ejecución de proyectos } \\
\text { estudiantiles. }\end{array}$ \\
\hline & & Apoyo a la promoción de semilleros. \\
\hline & & $\begin{array}{l}\text { Apoyo a la investigación en el aula } \\
\text { de clase. }\end{array}$ \\
\hline \multicolumn{3}{|c|}{ Brechas existentes } \\
\hline Adquisición (almacenamiento) & Difusión & Aplicación (creación) \\
\hline \multirow{2}{*}{$\begin{array}{l}\text { Se requiere de investigadores } \\
\text { expertos en metodologia de la } \\
\text { investigación y que tengan } \\
\text { interacción con otras comunidades } \\
\text { investigativas, no solamente } \\
\text { docentes que apenas se estén } \\
\text { formando, }\end{array}$} & & $\begin{array}{l}\text { Se detecta poco fomento por parte } \\
\text { de la Facultad al trabajo } \\
\text { colaborativo o en red (alianzas } \\
\text { estratégicas, convenios, } \\
\text { comunidades de práctica, } \\
\text { participación en gremios o } \\
\text { asociaciones, entre otras). }\end{array}$ \\
\hline & & $\begin{array}{l}\text { Se detecta poco fomento a la } \\
\text { internacionalización (movilidad, } \\
\text { intercambio de información). }\end{array}$ \\
\hline
\end{tabular}

Fuente: elaboración propia 


\section{Proceso clave gestión académica y administrativa}

La selección de los ítems de conocimiento que caracterizaron el proceso de gestión en el Programa de Derecho es: administración de unidades estratégicas; requisitos de acreditación de alta calidad; autoevaluación. Estos ítems provienen del análisis de la información suministrada por las fuentes consultadas en el estudio. Sin embargo, es preciso aclarar que la gestión académica y administrativa no se reconoce como una función sustantiva autónoma de la educación superior. Por el contrario, es un proceso que viene emergiendo en los últimos años, en la autoevaluación y el cumplimiento de lineamientos de alta calidad de las IES ${ }^{[14]}$.

Conviene destacar que, aunque hay administradores por cada proceso clave, en el proceso de docencia se tiene la estrategia de trabajar por áreas y cada cual cuenta con su director, pues la pretensión es que se conviertan en divisiones académico-administrativas y que estas gestionen el conocimiento. La tabla 5 muestra los factores de éxito y las brechas existentes encontradas en la gestión académica y administrativa del programa.

TABLA 5.

Gestión académica y administrativa

\begin{tabular}{|c|c|c|}
\hline \multicolumn{3}{|c|}{ Factores de éxito } \\
\hline Adquisición (almacenamiento) & Difusión & Aplicación (creación) \\
\hline $\begin{array}{l}\text { Gestión de las áreas documentada, plan de } \\
\text { desarrollo y plan de gestión. }\end{array}$ & Intranet & \multirow{2}{*}{ Coordinación del trabajo por áreas } \\
\hline \multirow{2}{*}{ Programa de Derecho acreditado } & $\begin{array}{l}\text { Plan de medios y prensa, blog de la } \\
\text { Facultad }\end{array}$ & \\
\hline & Programa de derecho acreditado & Programa de Derecho acreditado \\
\hline \multicolumn{3}{|c|}{ Brechas existentes } \\
\hline Adquisición (almacenamiento) & Difusión & Aplicación (creación) \\
\hline \multirow{3}{*}{$\begin{array}{l}\text { Uso reducido de repositorios } \\
\text { institucionales. }\end{array}$} & $\begin{array}{l}\text { Pocos eventos de socialización de } \\
\text { resultados de la gestión. }\end{array}$ & \\
\hline & $\begin{array}{l}\text { Faltan estrategias de socialización de las } \\
\text { politicas de alta calidad y el plan de } \\
\text { gestión y mejoramiento con toda la } \\
\text { comunidad académica. }\end{array}$ & \\
\hline & $\begin{array}{l}\text { Faltan estrategias de socialización del } \\
\text { modelo de autoevaluación con toda la } \\
\text { comunidad académica. }\end{array}$ & \\
\hline
\end{tabular}

\section{Etapa 7: El análisis de flujo de conocimiento}

El objetivo de esta etapa fue analizar cómo fluye el conocimiento en la organización.

Estos "activos" son analizados con respecto a los procesos de gestión del conocimiento propuestos por los autores de la metodología que se está siguiendo (adquisición, almacenamiento, aplicación, difusión y creación). La tabla 6 muestra el comportamiento del flujo, teniendo en cuenta personas, entidades, sistemas o documentos sobre los cuales fluye cada activo de conocimiento. 
TABLA 6.

Flujo del conocimiento en el Programa de Derecho de la Universidad de Manizales

\begin{tabular}{|c|c|c|c|c|c|}
\hline Procesos & $\begin{array}{c}\text { Ítem de } \\
\text { conocimiento (Tema } \\
\text { clave) }\end{array}$ & $\begin{array}{c}\text { Persona (s) que lo } \\
\text { poseen }\end{array}$ & $\begin{array}{c}\text { Entidades u } \\
\text { organizaciones que } \\
\text { lo poseen }\end{array}$ & $\begin{array}{c}\text { Sistema donde se } \\
\text { encuentra }\end{array}$ & $\begin{array}{l}\text { Documentos donde } \\
\text { se encuentra }\end{array}$ \\
\hline \multirow{5}{*}{ Proyección Social } & $\begin{array}{l}\text { Brigadas de } \\
\text { intervención a la } \\
\text { commidad }\end{array}$ & $\begin{array}{l}\text { Docentes asesores, } \\
\text { Estudalantes } \\
\text { practicantes, docenfes } \\
\text { onciliadores }\end{array}$ & & $\begin{array}{l}\text { Portal web de ka } \\
\text { facultad }\end{array}$ & \\
\hline & $\begin{array}{l}\text { Representación a } \\
\text { usuarios en litigio }\end{array}$ & $\begin{array}{l}\text { Estudantes } \\
\text { practicantes }\end{array}$ & Rama judicial & $\begin{array}{l}\text { Portal web de la rama } \\
\text { judicial }\end{array}$ & $\begin{array}{l}\text { Reglamento de } \\
\text { consultorio juridico, } \\
\text { expodientes de } \\
\text { representación }\end{array}$ \\
\hline & $\begin{array}{l}\text { Apdiencias de } \\
\text { concifación }\end{array}$ & Docentes concifadores & & $\begin{array}{l}\text { Portal web de la } \\
\text { facultad, SICAAC } \\
\text { (Mnjusticia) }\end{array}$ & $\begin{array}{l}\text { Manual de } \\
\text { competencias y } \\
\text { funciones del centro de } \\
\text { cocolación, archivos } \\
\text { del centro de } \\
\text { coecliación } \\
\end{array}$ \\
\hline & Cinica Juridica & $\begin{array}{l}\text { Asesores de clinica } \\
\text { juridica }\end{array}$ & & $\begin{array}{l}\text { Portal web de ka } \\
\text { facultad }\end{array}$ & \\
\hline & Euacación contimuada & $\begin{array}{l}\text { Docentes, externos } \\
\text { ponentes }\end{array}$ & Unversidades & $\begin{array}{l}\text { Portal web de la } \\
\text { facultad }\end{array}$ & Articulos, relatorias \\
\hline \multirow{3}{*}{ Investigación } & $\begin{array}{l}\text { Formación } \\
\text { investigativa }\end{array}$ & $\begin{array}{l}\text { Coordinadores de } \\
\text { semilero, Ideres de } \\
\text { grupo }\end{array}$ & $\begin{array}{l}\text { Colciencias, redes de } \\
\text { investigación }\end{array}$ & $\begin{array}{l}\text { Portal web de la } \\
\text { universidad }\end{array}$ & Resoluciôn rectoral \\
\hline & Imestigación ciestifica & $\begin{array}{l}\text { Docentes doctoresy } \\
\text { magister }\end{array}$ & Colciencias & $\begin{array}{l}\text { Portal web de } \\
\text { Colciencias, portal } \\
\text { webde la uriversidad }\end{array}$ & $\begin{array}{l}\text { Pubfcaciones, } \\
\text { reglementos de } \\
\text { investigaciones }\end{array}$ \\
\hline & $\begin{array}{l}\text { Metodologias de } \\
\text { investigación }\end{array}$ & $\begin{array}{l}\text { Docentes } \\
\text { investigadores }\end{array}$ & Redes de imestigación & $\begin{array}{l}\text { Portal web de ka } \\
\text { universidad }\end{array}$ & $\begin{array}{l}\text { Reglamentos de } \\
\text { investigaciones, Ifbros } \\
\text { sobre investigación } \\
\end{array}$ \\
\hline \multirow{3}{*}{ Docencia } & $\begin{array}{l}\text { Derecho sustantivo y } \\
\text { dogmático } \\
\text { (Conocimiento } \\
\text { disciplinar) }\end{array}$ & Docentes & Corte constitucional & $\begin{array}{l}\text { Bases de datos, portal } \\
\text { web de la universidad }\end{array}$ & $\begin{array}{l}\text { Cartas descriptivas, } \\
\text { microcurrículos, } \\
\text { publicaciones, códigos } \\
\text { de as áreas del } \\
\text { derecho } \\
\end{array}$ \\
\hline & $\begin{array}{l}\text { Metodologías de } \\
\text { enseñanza y el } \\
\text { aprendizaje } \\
\end{array}$ & $\begin{array}{l}\text { Docentes, expertos } \\
\text { externos, lideres } \\
\text { funcionales }\end{array}$ & $\begin{array}{l}\text { Ministerio de } \\
\text { educación, } \\
\text { universidades }\end{array}$ & $\begin{array}{l}\text { Portal web de ka } \\
\text { universidad }\end{array}$ & $\begin{array}{l}\text { PEP, PIF, } \\
\text { microcurriculos }\end{array}$ \\
\hline & $\begin{array}{l}\text { Derecho procesal y } \\
\text { procedimental } \\
\text { (Conocimiento } \\
\text { práctico) }\end{array}$ & $\begin{array}{l}\text { Docentes, expertos } \\
\text { externos }\end{array}$ & $\begin{array}{l}\text { Ministerio de justicia, } \\
\text { rama judicial }\end{array}$ & $\begin{array}{l}\text { Portal web de la rama } \\
\text { judicial, portal web del } \\
\text { ministerio de justicia, } \\
\text { portal web del senado } \\
\text { y portal web de la } \\
\text { universidad }\end{array}$ & $\begin{array}{l}\text { Códigos de } \\
\text { procedimiento, } \\
\text { expedientes, actas de } \\
\text { conciliación, } \\
\text { microcurriculos y } \\
\text { publicaciones }\end{array}$ \\
\hline \multirow{3}{*}{ Gestión } & $\begin{array}{l}\text { Administración de } \\
\text { unidades estratégicas }\end{array}$ & $\begin{array}{l}\text { Lideres funcionales, } \\
\text { decanatura }\end{array}$ & Universidades & $\begin{array}{l}\text { Portal web de la } \\
\text { universidad, SIGUM }\end{array}$ & $\begin{array}{l}\text { Reglamentos } \\
\text { institucionales, manual } \\
\text { de funciones y perfiles, } \\
\text { informes de gestión }\end{array}$ \\
\hline & $\begin{array}{l}\text { Requisitos de } \\
\text { acreditación de alta } \\
\text { calidad }\end{array}$ & $\begin{array}{l}\text { Lideres funcionales, } \\
\text { decanatura y docentes }\end{array}$ & $\begin{array}{l}\text { Ministerio de } \\
\text { educación, } \\
\text { universidades }\end{array}$ & $\begin{array}{l}\text { Portal web ministerio } \\
\text { de educación, portal } \\
\text { web de la universidad }\end{array}$ & $\begin{array}{l}\text { Plan de desarrollo, } \\
\text { PEP, modelo } \\
\text { pedagógico } \\
\text { institucional y } \\
\text { resolución de } \\
\text { acreditación en alta } \\
\text { calidad. }\end{array}$ \\
\hline & Autoevaluación & $\begin{array}{l}\text { Egresados, } \\
\text { estudiantes, lideres } \\
\text { funcionales, decanatura } \\
\text { y docentes }\end{array}$ & $\begin{array}{l}\text { Ministerio de } \\
\text { educación, } \\
\text { universidades, gremios } \\
\text { económicos y } \\
\text { cooperantes en } \\
\text { alianzas estratégicas. }\end{array}$ & $\begin{array}{l}\text { Portal web de la } \\
\text { universidad, portal } \\
\text { web del ministerio de } \\
\text { educación }\end{array}$ & $\begin{array}{l}\text { Modelo de } \\
\text { autoevaluación }\end{array}$ \\
\hline
\end{tabular}


En la tabla del flujo de conocimiento, se observa que este circula con eficiencia en el proceso de proyección social, especialmente en la labor que realiza el consultorio jurídico y del centro de conciliación. Probablemente favorece la vigilancia del Ministerio de Justicia y el Derecho, que exige que el programa tenga documentadas las asesorías jurídicas, las conciliaciones celebradas, las constancias de no acuerdo conciliatorio y de no comparecencia a las audiencias de conciliación, las representaciones judiciales a los usuarios, los repartos de expedientes, los rechazos de solicitudes de atención, entre otros. Inclusive, sumado a los archivos que debe tener el programa para conservar y custodiar los documentos relacionados, los administradores de este proceso están obligados a actualizar constantemente la página del Ministerio de Justicia en materia de conciliación. Por ello, es notoria la adecuada gestión del conocimiento en el proceso de proyección social con respecto a los otros tres procesos.

En el proceso de gestión académica y administrativa pasa algo similar, pues el programa y la facultad están vigilados por el Ministerio de Educación Nacional, quien establece los lineamientos en materia de educación superior y de acreditación de alta calidad, para los programas que tengan interés en lograr altos estándares de calidad de la formación, tal como le ocurre actualmente al Programa de Derecho que se encuentra acreditado desde 2012 y actualmente está en proceso de renovación de la acreditación.

La inspección que ejerce el Ministerio de Educación tiene influencia en el proceso de docencia por ser factor clave en el quehacer de la universidad. El proceso de investigación, especialmente la investigación científica, acoge los lineamientos de Colciencias ${ }^{[15]}$, que cuenta con la plataforma ScienTI donde se debe ingresar y actualizar la información como las hojas de vida de investigadores y la información de grupos de investigación ${ }^{[16]}$.

\section{Etapa 8: Mapa de conocimiento}

El objetivo de esta etapa fue el de representar el conocimiento organizacional. La elaboración del mapa se logró gracias a la información recolectada mediante el inventario y el flujo de conocimiento ${ }^{[17]}$, que dio la pauta sobre los ítems de conocimiento, quién los administra, cómo y a quién se distribuyen, y sobre su uso y apropiación. Con esta información se elaboró el mapa de conocimiento en el que se visualiza el conocimiento requerido, el creado, el proporcionado y sus usos, las personas que intercambian conocimiento y el conocimiento faltante ${ }^{[18]}$.

Este ejercicio implicó la valoración del conocimiento existente, que permite establecer los factores de éxito y las brechas de conocimiento. Para la medición se tomaron como referencia los siguientes rangos:

De 1\%-25\%: conocimiento incipiente. La gestión del conocimiento en el proceso clave no se considera eficiente, ya sea porque no se realiza ninguna actividad que permita una adecuada gestión o se cuenta con actividades aisladas sin un objetivo claro.

De 26\%-50\%: conocimiento regular. Significa que hay una actitud propositiva y consciente en gestión del conocimiento en el proceso, pero apenas se han iniciado actividades relacionadas sin un plan concreto o sin resultados comprobables.

De 51\%-75\%: conocimiento bueno. La gestión del conocimiento en el proceso clave es eficiente y continua, y se cuenta con un plan de trabajo de corto plazo.

De 76\%-100\%: conocimiento excelente. La gestión del conocimiento se considera estratégica para el proceso y se cuenta con un plan de trabajo de corto y largo plazo estructurado con actividades de seguimiento periódico, acciones de mantenimiento y de mejora continua. 
TABLA 7.

Mapa de conocimiento del Programa de Derecho de la Universidad de Manizales

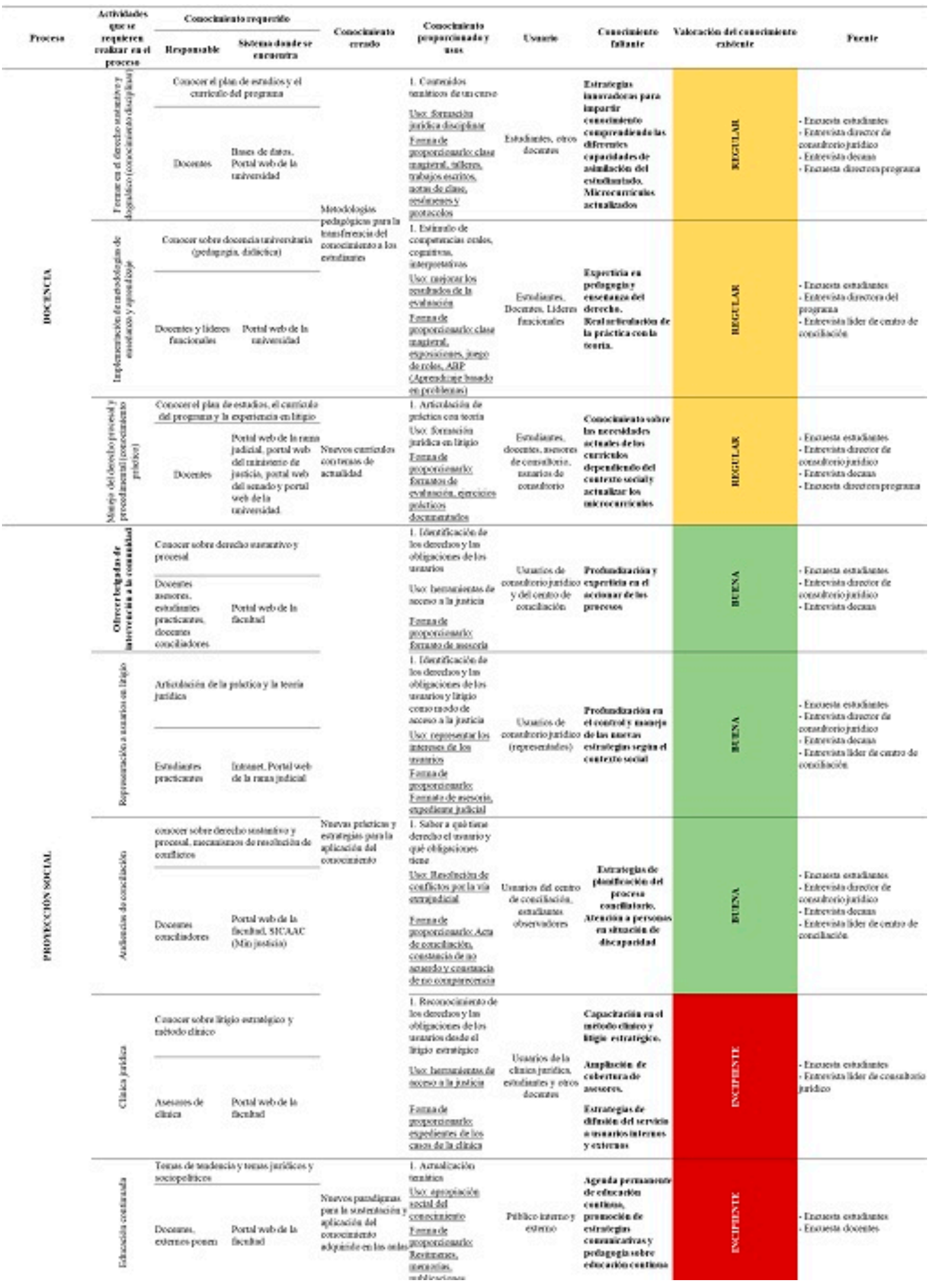

Fuente: elaboración propia 
TABLA 7.

Mapa de conocimiento del Programa de Derecho de la Universidad de Manizales (Cont.)

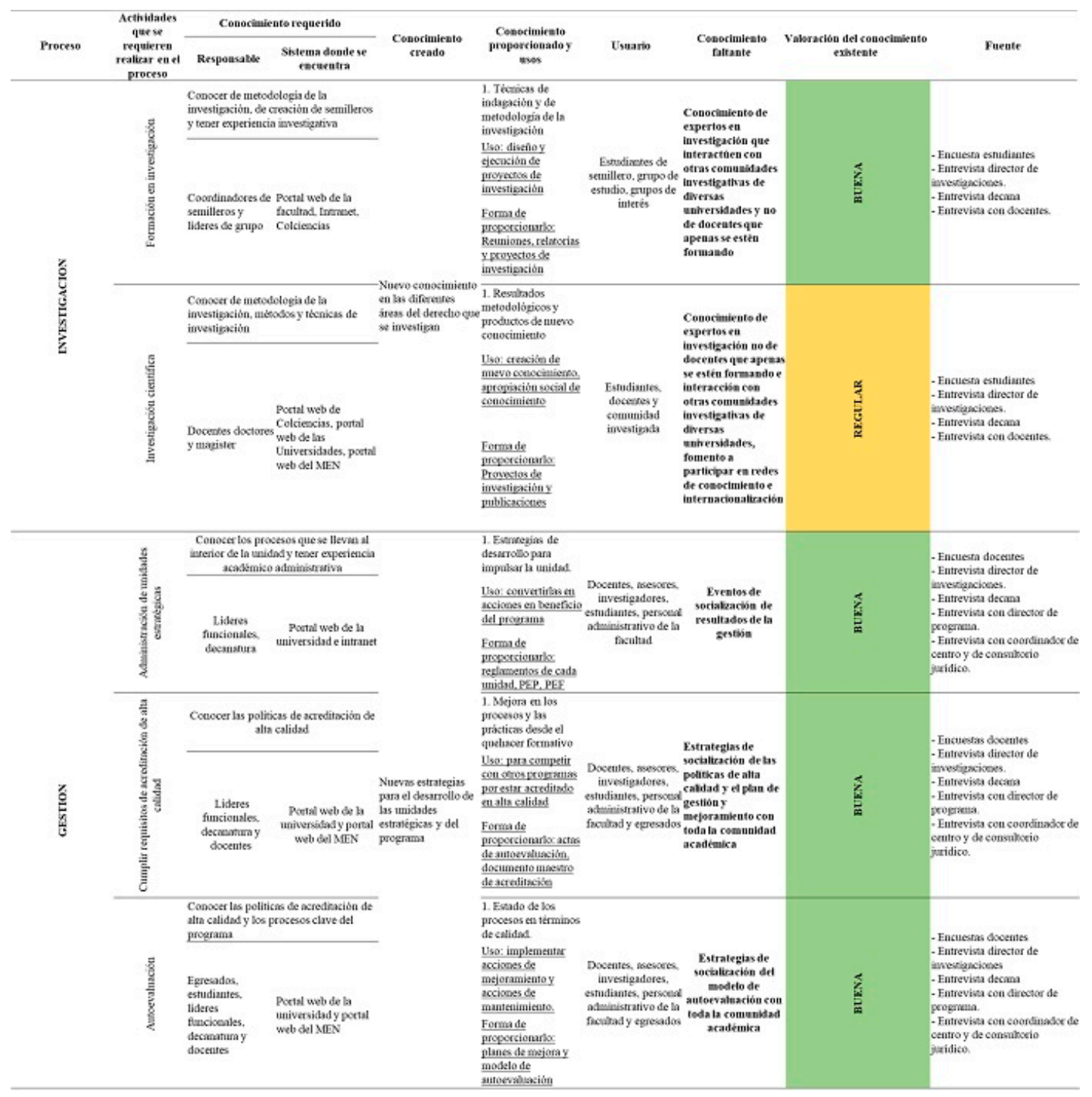

Fuente: elaboración propia

\section{Conclusiones, recomendaciones y limitaciones}

Tras la realización de este estúdio, se concluye de manera general que:

- Es preciso reconocer que, en una organización como la Universidad de Manizales, cuyo producto es el conocimiento, cumple un rol clave la gestión del conocimiento en el desarrollo del programa de Derecho, puesto que, a través de esta, se pueden propiciar ambientes que permitan el flujo constante del conocimiento, siendo este el activo principal.

- Aunque la Facultad de Ciencias Jurídicas tiene claro el propósito del servicio que ofrece, aún tiene mucho camino por recorrer con respecto a temas de actualización que corresponden a propuestas innovadoras para el mejoramiento del programa, empezando por la sensibilización de todos los actores que intervienen en sus procesos. Se identificó que el conocimiento clave puede perderse cuando los docentes o el personal administrativo se retiran de la facultad, esto lo demuestra la proporción de docentes que indican que no se documenta el conocimiento valioso y que, además, se percibe resistencia para compartirlo.

- Se observa coherencia entre los documentos base del programa y los de la institución con respecto a las labores que se proponen en los procesos. Sin embargo, no se muestra una capacidad instalada que pueda soportar las pretensiones del programa sin importar la intención que este tenga para su crecimiento y desarrollo.

- El programa de derecho busca alternativas implícitas en el quehacer diario para satisfacer la necesidad de transformaciones innovadoras del desarrollo de los procesos. De manera que en el momento en que la facultad se proponga usar la gestión del 
conocimiento para incrementar la propuesta de valor en su servicio, la formación de estrategias innovadoras pueda mejorar sus niveles de competencia frente a los demás programas en el ámbito nacional e internacional.

- Se confirma la necesidad de que todas las IES implementen auditorías de conocimiento con el fin de optimizar la administración de su activo más preciado: el conocimiento.

- Aplicar una auditoría de conocimiento se ha vuelto una herramienta clave para identificar las relaciones que existen en la transferencia de conocimiento y cómo fluye en medio de los procesos de la organización, lo que permite identificar oportunidades de mejora y hacer propuestas para resarcir las falencias que se encuentren en términos de gestión del conocimiento.

- El programa de derecho de la Universidad de Manizales busca alternativas para conseguir nuevo conocimiento y aplicarlo en la sociedad a través del fomento de la investigación, lo cual ha llevado al programa a la acreditación de alta calidad avalada por el Ministerio de Educación Nacional y, por lo tanto, se observa la receptividad hacia nuevas propuestas. Esto es un comienzo para considerar un plan que les permita gestionar el conocimiento de una manera consciente y ordenada.

- La facultad cuenta con una planta docente joven, lo que permite que lleguen perspectivas frescas y nuevas ideas. Sin embargo, se deben abrir espacios para apoyar la adquisición de conocimiento experto que ayude a los docentes jóvenes a desempeñar una labor con mayor soporte para el desarrollo de sus actividades. Por esto, el conocimiento debe ser de fácil acceso y debe estar bien documentado y almacenado, para que se dé una transferencia efectiva.

Además, frente a los objetivos contemplados en el estudio, se tiene lo siguiente:

Con relación al primer objetivo específico - Seleccionar la metodología de auditoría de conocimiento que sea idónea para la evaluación del estado de la gestión del conocimiento en la docencia, la investigación, la proyección social y la gestión en el Programa de Derecho de la Facultad de Ciencias Jurídicas de la Universidad de Manizales-, se concluye que la metodología que mejor puede aplicarse es aquella que, además de brindar un panorama integral para establecer el estado de la gestión del conocimiento, haya sido antes validada al interior de otros programas educativos en instituciones de educación superior. Luego de ser comparada con otros modelos y metodologías al respecto, se seleccionó la propuesta por Pérez-Soltero et al. ${ }^{[19]}$, la cuál fue validada a través de un proyecto de investigación en la Universidad Veracruzana, región Xalapa.

Sobre el segundo objetivo específico - Establecer el estado de la gestión del conocimiento en el Programa de Derecho de la Facultad de Ciencias Jurídicas de la Universidad de Manizales-, se concluye que no existe un sistema formal de gestión del conocimiento y aunque en el quehacer diario de los docentes del programa se realizan actividades para gestionar el conocimiento, en general, el estado de los procesos clave que esta implica (adquisición, difusión y aplicación) presenta un nivel medio. Por otro lado, el desarrollo de esta investigación permitió reconocer los principales activos de conocimiento alrededor de los procesos clave que permiten impulsar la innovación en el programa de derecho, y en ello se fundamenta el plan de acción construido.

Frente al tercer objetivo específico - Determinar los factores críticos de éxito y las brechas de mejora en la gestión del conocimiento en el Programa de Derecho de la Facultad de Ciencias Jurídicas de la Universidad de Manizales-, se concluye que entre los principales factores de éxito están, en primer lugar, la alineación entre las políticas institucionales y la gestión administrativa del programa, con lo cual se tiene un enfoque estratégico y funcional claro que determina la pertinencia, utilidad y aporte del conocimiento que se tiene, que se requiere o que se debe gestionar a futuro. En segundo lugar, la existencia de escenarios y espacios que promueven la comunicación directa y el intercambio de conocimiento entre los diversos actores ligados al programa.

Por su parte, las principales brechas identificadas son la poca experiencia de un grupo de docentes importante del programa que aún tienen carencias en la formación sobre algunos procesos críticos, en especial en materia de investigación. A esto se suma el escaso uso de las TIC para documentar y compartir el conocimiento dentro y fuera de la institución.

Con relación al objetivo general —Construir un plan de acción para optimizar la gestión del conocimiento como insumo básico de la innovación educativa en el Programa de Derecho de la Facultad de Ciencias Jurídicas de la Universidad de Manizales-, se propone un plan de acción orientado tanto a fortalecer los factores de éxito como a cerrar las brechas identificadas en cada proceso clave, soportado en un esquema de tiempo de realización de actividades y desde una perspectiva sistémica, en tanto se propende que se conecten 
entre ellas y permitan el desarrollo de una estrategia de gestión del conocimiento ordenada. Dicho plan incluye tanto los procesos clave identificados en la auditoría realizada, como los principales actores que en ellos intervienen.

Así las cosas, el presente trabajo aporta una metodología concreta de auditoría del conocimiento y evidencia empírica de cómo utilizarla para construir un plan de acción, que bien pueden servir como base para futuras investigaciones sobre la gestión del conocimiento en las organizaciones en general, y en las IES en particular.

Finalmente, por tratarse de un estudio de caso los resultados de la investigación son válidos solamente para el Programa de Derecho de la Facultad de Ciencias Jurídicas de la Universidad de Manizales y, por tanto, no son generalizables. Adicionalmente, aunque el instrumento de auditoría del conocimiento utilizado ya ha sido validado en otros estudios, puede requerir ajustes según el tipo de organizaciones en los que se pretenda aplicar o en el caso de pretender abordar un sector de actividad económica de manera conjunta.

\section{Referencias}

Alonso Pérez-Soltero, Mario Barcelo-Valenzuela, Gerardo Sánchez-Schmitz, Rene Navarro-Hernandez \& CarlosArturo Torres Gastelu. Diseño de una ontología para la reutilización del conocimiento en los procesos de auditoría del conocimiento. 3 Memorias de la Séptima Conferencia Iberoamericana de Sistemas, Cibernética e Informática, CISCI, 164-169 (2008).

Augusto Pérez-Lindo, Principios y aplicaciones de la gestión del conocimiento en la Universidad, en Aportes de las ciencias sociales y humanas al análisis de la problemática universitaria, Ed. Prometeo, 67-76 (2008).

Alonso Pérez-Soltero. La Auditoria del conocimiento en las organizaciones. Revista Universidad de Sonora, n. ${ }^{\circ}$ 25, 25-28. (2009). http://www.revistauniversidad.uson.mx/revistas/25-7.pdf

Alonso Pérez-Soltero, Mario Barcelo-Valenzuela, Gerardo Sánzhez-Schmitz, Fernando Martin-Rubio, Jose Tomas Palma-Méndez, \& Adolfo Alberto Vanti, A Model and Methodology to Knowledge Auditing Considering Core Processes, 5 The Icfai Journal of Knowledge Management, n. ${ }^{\circ} 1,18$ (2007).

Begoña Gros Salvat \& Pablo Lara Navarra, Estrategias de innovación en la educación superior: El caso de la Universitat Oberta de Catalunya, Revista Iberoamericana de Educación, n. ${ }^{\circ} 49,223-245$ (2009).

Carlos Molina, María Carreño, Rafaela Sayas, Mónica Montoya, Denisse Herreño, Yadira Alarcón \& José Alvarez. Diagnóstico y lineamientos técnicos para los distintos escenarios de la práctica jurídica en los programas de Derecho de las Instituciones de Educación Superior en Colombia. Ed. Acofade. (2014).

Cristhian Guillermo Naranjo, Prácticas de recursos humanos y gestión del conocimiento en las organizaciones: una mirada desde las dimensiones del trabajador del conocimiento, 19 Ánfora, n. ${ }^{\circ} 33,115-142$. (2012).

Colciencias, Modelo de medición de grupos de investigación, desarrollo tecnológico o de innovación y de reconocimiento de investigadores del sistema nacional de ciencia, tecnología e innovación. Dirección de Fomento a la Investigación (2014)

Chun Wei Choo, La organización inteligente, el empleo de la información para dar significado, crear conocimiento y tomar decisiones, Oxford University Press (1999).

Ernesto Galvis \& Jenny Marcela Sánche, Evaluación de la gestión del conocimiento: Una revisión sistemática de literatura, 15 Tendencias, n. ${ }^{\circ}$ 2, 151-170 (2014).

Facultad de Ciencias Jurídicas, Proyecto Educativo del Programa. Universidad de Manizales (2015).

Gerardo Arceo, El impacto de la gestión del conocimiento y las tecnologias de información en la innovación: un estudio en las pymes del sector agroalimentario de Cataluña. Barcelona (2009) (tesis doctoral, Universitat Politècnica de Catalunya).

Isabel García, Estado de la Cuestión de la auditoría de la información como metodología para la optimización del conocimiento en las organizaciones (2008) (Tesis de maestría, Universidad Politécnica de Valencia).

Ikujiro Nonaka, A Dynamic Theory of Organizational Knowledge Creation, 5 Organization Science, n. ${ }^{\circ}$ 1, 14-37 (1994). 
Julio César Flores, La Gestión del conocimiento y las herramientas colaborativas: una alternativa de aplicación en Instituciones de educación superior, 71 Revista de investigación, n. ${ }^{\circ}$ 34, 11-32 (2010).

Jair Alexis Galvis Pérez, Mapas de conocimiento como una herramienta de apoyo para la gestión del conocimiento, CINTEL \& InteracTIC (2009).

Juan Carlos Mijangos \& Karla Sugey Manzo, Gestión del conocimiento de tres cuerpos académicos con solidados del área educativa, Sinéctica, n. ${ }^{\circ} 38,1-13$ (2012).

Ley 1188 de 2008. Por la cual se regula el registro calificado de programas de educación superior y se dictan otras disposiciones. 25 de abril de 2008. D.O. n. ${ }^{\circ} 46971$.

Ley 1286 de 2009. Por la cual se modifica la Ley 29 de 1990, se transforma a Colciencias en Departamento Administrativo, se fortalece el Sistema Nacional de Ciencia, Tecnología e Innovación en Colombia y se dictan otras disposiciones. 23 de enero de 2009. D. O. n. 47241.

María Teresa Baquerin-de-Riccitelli, El impacto de la comunidad en las brechas del conocimiento, 20 Comunicación Y Sociedad, n. ${ }^{\circ}$ 1, 9-27 (2007).

María Dolores Gil, Alonso Pérez-Sotero, \& Gilberto López Orozco, La auditoría del conocimiento como etapa previa a la gestión del conocimiento en una institución educativa mexicana, en VI Congreso internacional de análisis organizacional. México: Organización del Congreso (2008).

Sandra Duque, Marta Lucía Quintero, \& Derfrey Antoio Duque, Sobre una conceptualización de la popularización del Derecho en la educación superior, Zona Próxima, n. 21 (2014). http://dx.doi.org/10.14482/zp.21.6030

Yeter Caraballo, Dianelis Mesa \& J. Herrera, Herramientas de gestión del conocimiento: convergencias hacia un aprendizaje organización, 43 Revista Cubana de Ciencia Agrícola, n. ${ }^{\circ}$ 1, 1-13 (2009).

\section{Notas}

* Artículo de investigación. Producto de dos proyectos de investigación. El primero, "Gestión del conocimiento en el marco de la innovación en el Programa de Derecho de la Facultad de Ciencias Jurídicas de la Universidad de Manizales: plan de acción” fue ejecutado durante el año 2016 por el primer autor, con el fin de obtener el título de magíster en gerencia de la innovación y el conocimiento, ofrecido por la Universidad EAFIT de Medellín. El segundo proyecto, que se titula "Procesos académicos y administrativos en los programas de derecho e ingeniería de la Universidad de Manizales: propuesta de mejora en el marco de la innovación y la gestión del conocimiento", fue ejecutado durante el año 2017, con financiación de la Universidad de Manizales, donde los dos autores fungen como asesores y coinvestigadores.

[1] Está en manos de las directivas de la institución, decidir sobre la viabilidad del plan en términos de destinación o adquisición de los recursos requeridos para su implementación.

[2] Chun Wei Choo, La organización inteligente, el empleo de la información para dar significado, crear conocimiento y tomar decisiones, Oxford University Press (1999); Isabel García, Estado de la Cuestión de la auditoría de la información como metodología para la optimización del conocimiento en las organizaciones (2008) (Tesis de maestría, Universidad Politécnica de Valencia).

[3] Yeter Caraballo, Dianelis Mesa \& J. Herrera, Herramientas de gestión del conocimiento: convergencias hacia un aprendizaje organización, 43 Revista Cubana de Ciencia Agrícola, n. ${ }^{\circ}$ 1, 1-13 (2009); Cristhian Guillermo Naranjo, Prácticas de recursos humanos y gestión del conocimiento en las organizaciones: una mirada desde las dimensiones del trabajador del conocimiento, 19 Ánfora, n. 33, 115-142. (2012); Ernesto Galvis \& Jenny Marcela Sánche, Evaluación de la gestión del conocimiento: Una revisión sistemática de literatura, 15 Tendencias, n. 2, 151-170 (2014); Julio César Flores, La Gestión del conocimiento y las herramientas colaborativas: una alternativa de aplicación en Instituciones de educación superior, 71 Revista de investigación, n. ${ }^{\circ}$ 34, 11-32 (2010); Juan Carlos Mijangos \& Karla Sugey Manzo, Gestión del conocimiento de tres cuerpos académicos con solidados del área educativa, Sinéctica, n. ${ }^{\circ} 38,1-13$ (2012).

[4] Gerardo Arceo, El impacto de la gestión del conocimiento y las tecnologías de información en la innovación: un estudio en las pymes del sector agroalimentario de Cataluña. Barcelona (2009) (tesis doctoral, Universitat Politècnica de Catalunya); María Teresa Baquerin-de-Riccitelli, El impacto de la comunidad en las brechas del conocimiento, 20 Comunicación Y Sociedad, n. ${ }^{\circ}$ 1, 9-27 (2007). 
[5] Vease Augusto Pérez-Lindo, Principios y aplicaciones de la gestión del conocimiento en la Universidad, en Aportes de las ciencias sociales y humanas al análisis de la problemática universitaria, Ed. Prometeo, 67-76 (2008).

[6] Vease Alonso Pérez-Soltero. La Auditoría del conocimiento en las organizaciones. Revista Universidad de Sonora, n. ${ }^{\circ} 25$, 25-28. (2009).

[7] Ley 1188 de 2008. Por la cual se regula el registro calificado de programas de educación superior y se dictan otras disposiciones. 25 de abril de 2008. D.O. n. ${ }^{\circ} 6971$.

[8] Alonso Pérez-Soltero, Mario Barcelo-Valenzuela, Gerardo Sánchez-Schmitz, Rene Navarro-Hernandez \& CarlosArturo Torres Gastelu. Diseño de una ontología para la reutilización del conocimiento en los procesos de auditoría del conocimiento. 3 Memorias de la Séptima Conferencia Iberoamericana de Sistemas, Cibernética e Informática, CISCI, 164-169 (2008).

[9] Begoña Gros Salvat \& Pablo Lara Navarra, Estrategias de innovación en la educación superior: El caso de la Universitat Oberta de Catalunya, Revista Iberoamericana de Educación, n. ${ }^{\circ}$ 49, 227 (2009).

[10] Gryna, citado por Alonso Pérez-Soltero, Mario Barcelo-Valenzuela, Gerardo Sánchez-Schmitz, Rene Navarro-Hernandez \& CarlosArturo Torres Gastelu. Diseño de una ontología para la reutilización del conocimiento en los procesos de auditoría del conocimiento. 3 Memorias de la Séptima Conferencia Iberoamericana de Sistemas, Cibernética e Informática, CISCI, 164-169 (2008).

[11] Íd.

[12] Sandra Duque, Marta Lucía Quintero, \& Derfrey Antoio Duque, Sobre una conceptualización de la popularización del Derecho en la educación superior, Zona Próxima, n. 21 (2014).

[13] Ikujiro Nonaka, A Dynamic Theory of Organizational Knowledge Creation, 5 Organization Science, n. ${ }^{\circ}$ 1, 14-37 (1994).

[14] Carlos Molina, María Carreño, Rafaela Sayas, Mónica Montoya, Denisse Herreño, Yadira Alarcón \& José Alvarez. Diagnóstico y lineamientos técnicos para los distintos escenarios de la práctica jurídica en los programas de Derecho de las Instituciones de Educación Superior en Colombia. Ed. Acofade. (2014).

[15] Ley 1286 de 2009. Por la cual se modifica la Ley 29 de 1990, se transforma a Colciencias en Departamento Administrativo, se fortalece el Sistema Nacional de Ciencia, Tecnología e Innovación en Colombia y se dictan otras disposiciones. 23 de enero de 2009. D. O. n. ${ }^{\circ} 47241$.

[16] Colciencias, Modelo de medición de grupos de investigación, desarrollo tecnológico o de innovación y de reconocimiento de investigadores del sistema nacional de ciencia, tecnología e innovación. Dirección de Fomento a la Investigación (2014)

[17] Véase: Jair Alexis Galvis Pérez, Mapas de conocimiento como una herramienta de apoyo para la gestión del conocimiento, CINTEL \& InteracTIC (2009).

[18] Alonso Pérez-Soltero, Mario Barcelo-Valenzuela, Gerardo Sánzhez-Schmitz, Fernando Martin-Rubio, Jose Tomas PalmaMéndez, \& Adolfo Alberto Vanti, A Model and Methodology to Knowledge Auditing Considering Core Processes, 5 The Icfai Journal of Knowledge Management, n. ${ }^{\circ} 1,18$ (2007).

[19] María Dolores Gil, Alonso Pérez-Sotero, \& Gilberto López Orozco, La auditoría del conocimiento como etapa previa a la gestión del conocimiento en una institución educativa mexicana, en VI Congreso internacional de análisis organizacional. México: Organización del Congreso (2008).

\section{Licencia Creative Commons CC BY 4.0}

Para citar este artículo/To cite this article: Camilo González Carreño \& Monica Cecilia Montoya Escobar, Gestión del conocimiento en el marco de la innovación en la Facultad de Ciencias Juridicas de la Universidad de Manizales: Auditoria de conocimiento, 68 Vniversitas, n. ${ }^{\circ} 139$ (2019). https://doi.org/10.11144/Javeria na.vj139.gcmi 